\title{
Phenotypic and functional characterisation of myofibroblasts, macrophages, and lymphocytes migrating out of the human gastric lamina propria following the loss of epithelial cells
}

\author{
K C Wu, L M Jackson, A M Galvin, T Gray, C J Hawkey, Y R Mahida
}

\begin{abstract}
Background-The basement membrane of human colonic mucosa contains numerous discrete pores. We have recently shown that following loss of the surface epithelium, many cells migrate out of the colonic lamina propria via basement membrane pores.

Aims-To characterise cells migrating out via basement membrane pores of the human gastric lamina propria, following loss of the surface epithelium.

Methods-Fresh human gastric mucosal samples were completely denuded of epithelial cells and placed in culture. Tissue samples were studied by electron microscopy (EM) and cells by EM, FACS analysis, immunohistochemistry, and reverse transcription polymerase chain reaction (RT-PCR).

Results-EM showed numerous discrete pores (0.65-8.29 $\mu \mathrm{m}$ in diameter) in the subepithelial basement membrane. During culture of mucosal samples denuded of epithelial cells, lymphocytes, macrophages, and myofibroblasts migrated out of the lamina propria via the basement membrane pores. The lymphocytes were predominantly CD45RO+ and CD69+ T cells. Macrophages were shown to express cyclooxygenase (COX) 1 and 2 enzymes. Myofibroblasts were established in culture and, despite prolonged culture and passage, retained their phenotype. They expressed mRNA and protein for COX 1 and 2 enzymes and their release of prostaglandin $\mathrm{E}_{2}$ was inhibited by selective COX 1 and 2 inhibitors.

Conclusions-Lamina propria cells migrating out of cultured denuded gastric mucosal samples have been characterised phenotypically and functionally. Such cells would be suitable for studies of their interactions with epithelial cells and also with Helicobacter pylori and its products. (Gut 1999;44:323-330)
\end{abstract}

Keywords: stomach; basement membrane; lymphocytes; macrophages; myofibroblasts

The gastric mucosa is lined by a monolayer of columnar epithelial cells, separated from the lamina propria by a basement membrane. The normal lamina propria consists of connective tissue matrix within which lie many different cell types, $\mathrm{T}$ and $\mathrm{B}$ lymphocytes, plasma cells, macrophages, myofibroblasts, and cells that make up blood and lymphatic vessels. During inflammation, polymorphonuclear cells, lymphocytes, and monocytes are recruited into the lamina propria from the systemic circulation. The deepest layers of the gastric mucosa are formed by the muscularis mucosae, which consists of a layer of smooth muscle cells, and the submucosa below it. The latter consists almost exclusively of connective tissue. In recent years, there has been increasing appreciation of the importance of interactions between the epithelial monolayer and cellular components of the lamina propria in mucosal physiology and pathophysiology. ${ }^{12}$ Cells within the lamina propria that may interact with the epithelial cells include lymphocytes, macrophages, and myofibroblasts. Some of these interactions may occur via lamina propria cell derived products of the two isoforms of the cyclooxygenase (COX) enzyme, COX 1 and $2 .^{3}$ These enzymes catalyse the initial steps in the formation of prostaglandins from arachidonic acid.

In the mucosa of the large intestine, distinct pores are present in the basement membrane, through which cells are capable of migrating and interacting with epithelial cells. ${ }^{45}$ We have recently shown that, following the loss of epithelial cells, large numbers of colonic mucosal lymphocytes, macrophages, eosinophils, and myofibroblasts migrate out of the lamina propria via basement membrane pores. ${ }^{56}$

In this study, we applied similar techniques to the gastric mucosa. The aim of the study was to characterise the lamina propria cells migrating through subepithelial basement membrane pores of human gastric mucosa, following the loss of the surface epithelium. We show by electron microscopy that numerous discrete pores are present in the basement membrane of gastric mucosal samples. During culture of gastric mucosal samples denuded of epithelial cells, lymphocytes, macrophages, and myofibroblasts migrate out of the lamina propria, via

Abbreviations used in this paper: $\mathrm{COX}$, cyclooxygenase; EM, electron microscopy; RT-PCR, reverse transcription polymerase chain reaction; HBSS, Hanks's balanced salt solution; FCS, fetal calf serum; FACS, fluorescein activated cell sorter; DMEM, Dulbecco's modified Eagle's medium; HRP, horseradish peroxidase; DAB, diaminobenzidine; FITC, fluorescein isothiocyanate; PE, phytoerythrin; GAPDH, glyceraldehyde-3-phosphate dehydrogenase.
Dr Mahida.

Accepted for publication 15 October 1998 
pores in the basement membrane. Myofibroblasts were established in pure culture and, together with macrophages, shown to express COX 1 and 2 enzymes.

\section{Materials and methods}

MUCOSAL TISSUE AND CELLS

Normal mucosal samples were obtained from fresh human gastric resection specimens (resected for tumour; $n=11$ ). Mucosa samples, which were histologically normal, were taken at least $5 \mathrm{~cm}$ away from the tumour. Epithelial cells were removed from the basement membrane of mucosal strips using a previously described technique ${ }^{5}$ with modifications. In brief, after 15 minutes' incubation in $1 \mathrm{mmol} / 1$ dithiothreitol (Sigma, St Louis, Missouri, USA), mucosal samples were denuded of epithelial cells by five sequential 30 minute incubations in $1 \mathrm{mmol} / \mathrm{l}$ EDTA (Sigma). Between each incubation with EDTA, the mucosal samples were washed with calcium and magnesium free Hanks's balanced salt solution (HBSS; GIBCO/BRL, Gaithersburg, Maryland, USA). The de-epithelialised mucosal samples were subsequently cultured at $37^{\circ} \mathrm{C}$ in RPMI 1640 containing $10 \%$ fetal calf serum (FCS; GIBCO/BRL). During culture, cells migrated out of the lamina propria matrix and appeared in suspension or adherent to the bottom of tissue culture dish. These cells were collected after three consecutive 24 hour periods of culture of the denuded mucosal samples, as previously described. ${ }^{5}$ The phenotype of these cells was investigated by immunohistochemistry (of cytospin preparations) and by fluorescein activated cell sorter (FACS; see below) analysis. The denuded mucosal samples were subsequently maintained in culture for up to six weeks, with change of medium every 72 hours.

In some studies, cells that migrated out of the lamina propria over the first 24 hours were cultured with opsonised zymosan. The latter was prepared as previously described. ${ }^{7}$ In brief, zymosan was incubated with fresh serum at $37^{\circ} \mathrm{C}$ for 30 minutes. It was then washed and incubated (at a final concentration of 15 $\mathrm{mg} / \mathrm{ml}$ ) for 60 minutes at $37^{\circ} \mathrm{C}$, with lamina propria cells $\left(2 \times 10^{6} / \mathrm{ml}\right)$. Cytospin preparations of cells were subsequently fixed in acetone and stored at $-70^{\circ} \mathrm{C}$ until used for immunohistochemistry (see below).

Established colonies of myofibroblasts were cultured in Dulbecco's modified Eagle's medium (DMEM; Sigma) supplemented with $10 \%$ FCS and $1 \%$ non-essential amino acids (NEAA; GIBCO/BRL). At confluence, the cells were passaged using $0.1 \%$ trypsin- $0.2 \%$ EDTA in a $1: 2$ to $1: 3$ split ratio. For immunocytochemistry, the cells were grown on glass coverslips and fixed in 10\% buffered formalin.

\section{IMMUNOCYTOCHEMISTRY}

Cells in cytospin preparations and those on glass coverslips were labelled with antibodies using the Vectastain ABC peroxidase kit (Vector Laboratories, Burlingame, California, USA). Antibodies used were: mouse monoclonal antibodies to CD3, CD19, CD68 (from
Dako, High Wycombe, UK), $\alpha$ smooth muscle actin, vimentin, and desmin (from Sigma) and polyclonal antibodies (rabbit) to cyclooxygenase 1 and 2 (COX-1 and COX-2, both from Cayman Chemical Co., Ann Arbor, Michigan, USA). Endogenous peroxidase activity was blocked with $6 \% \mathrm{H}_{2} \mathrm{O}_{2}$ in methanol before the cells were incubated at $4^{\circ} \mathrm{C}$ overnight with the primary antibodies. Biotinylated goat antimouse or antirabbit IgG was applied followed by avidin-biotinylated horseradish peroxidase (HRP) complex. Peroxidase activity in the latter was developed with diaminobenzidine (DAB).

The proportion of positively stained cells in each cytospin preparation was determined by analysing at least 200 cells. Cytospins were also stained with toluidine blue to determine the proportion of eosinophils present.

FACS ANALYSIS

Lamina propria cells were labelled with fluorescein isothiocyanate (FITC) or phytoerythrin (PE) conjugated mouse monoclonal antibodies and analysed by FACS as previously described. $^{8}$

The following antibody pairs were used: Hle-1/Leu-M3 (CD45/CD14), Leu-4/Leu-12 (CD3/CD19), Leu-3a/Leu-2a (CD4/CD8), and Leu-4/ (CD3/CD16/CD56) (all from Becton Dickinson). Individual FITC or PE labelled monoclonal antibodies were to CD3, CD25, CD69, CD45RO, and CD45RA (all from Dako)

\section{ELECTRON MICROSCOPY}

Denuded mucosal samples (before and after culture) and monolayers of myofibroblasts were fixed by immersion in $2.5 \%$ gluteraldehyde (in $0.1 \mathrm{~mol} / 1$ cacodylate buffer, $\mathrm{pH} 7.4$ ) for two hours. Subsequent processing was performed as previously described. ${ }^{9}$ Ultrathin (18 $\mathrm{nm}$ ) sections were mounted on copper grids, stained with uranyl acetate and lead citrate, and studied by using a Jeol 1200 EX transmission electron microscope (Jeol, Welwyn Garden City, UK).

REVERSE TRANSCRIPTION POLYMERASE CHAIN REACTION

RNA was isolated from the primary gastric myofibroblasts at passage 3 using RNAzol B (Biogenesis, Poole, UK) and cDNA synthesised by incubation of $10 \mu \mathrm{g}$ total RNA with random hexamer primer (Pharmacia Biotech) and Moloney murine leukaemia virus reverse transcriptase (RT; GIBCO/BRL). The cDNA was amplified by the polymerase chain reaction (PCR) using Taq polymerase (Promega, Madison, Wisconsin, USA) and the following primer pairs (synthesised based on published nucleotide sequences ${ }^{10}{ }^{11}$ ): $5^{\prime}$-GAG TCT TTC TCC AAC GTG AGC-3' (sense) and 5'-ACC TGG TAC TTG AGT TTC CCA-3' (antisense) to amplify a 350 base pair COX-1 product; 5'-TGA AAC CCA CTC CAA ACA CAG-3' (sense) and 5'-TCA TCA GGC ACA GGA GGA AG-3' (antisense) to amplify a 232 base pair COX-2 product; 5'-CCA CCC ATG GCA AAT TCC ATG GCA-3' (sense) and 


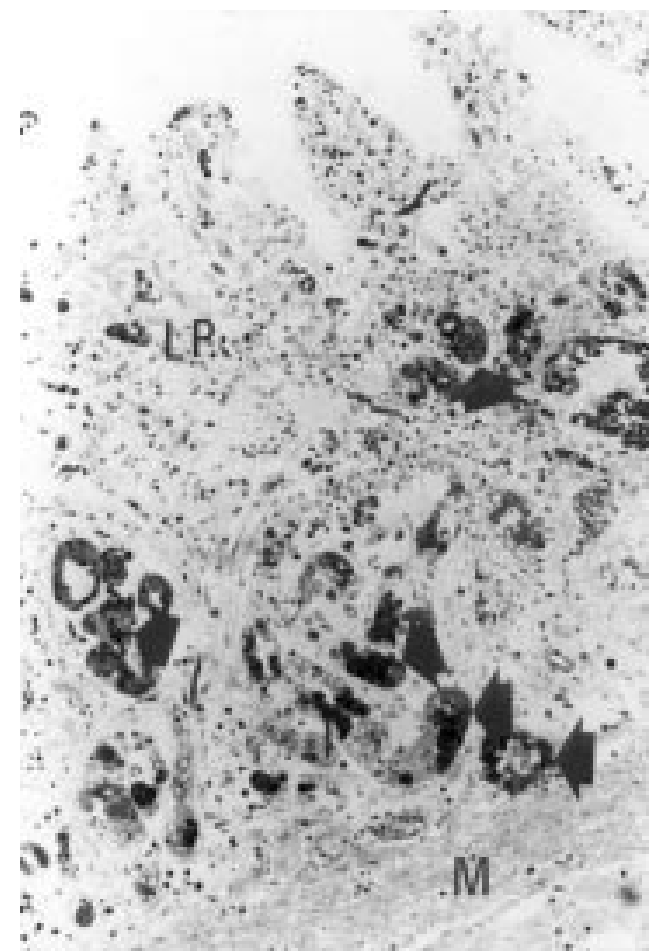

Figure 1 Photomicrograph of a toluidine blue stained section of a normal gastric mucosal sample, following three sequential incubations in EDTA. The surface epithelial cells have been removed to leave an intact lamina propria (LP). However, many epithelial cells remain in the glands (arrows) and in subsequent studies, they were removed following two further treatments with EDTA. M, muscularis mucosae.

5'-TCT AGA CGG CAG GTC AGG TCC ACC-3' (antisense; Stratagene, La Jolla, California, USA) to amplify a 600 base pair and human glyceraldehyde-3-phosphate dehydrogenase (GAPDH) product. Amplification was performed using 30 PCR cycles, which consisted of denaturation at $95^{\circ} \mathrm{C}$ for 45 seconds, annealing at $54^{\circ} \mathrm{C}$ for 90 seconds, and extension at $72^{\circ} \mathrm{C}$ for 90 seconds, and completed by elongation at $72^{\circ} \mathrm{C}$ for three minutes. PCR reaction products were separated on a $1.5 \%$ agarose gel and visualised by ethidium bromide. The specificities of RTPCR for COX-1, COX-2, and GAPDH have previously been confirmed in our laboratory.

PROSTAGLANDIN (PG) E 2 PRODUCTION

Gastric myofibroblasts were grown to confluence in 24 well plates (Nunc, GIBCO/BRL) and initially preincubated in medium containing control buffer or a specific COX-1 inhibitor (SC-58560, gift from Searle, Skokie, Illinois, USA) or a specific COX-2 inhibitor (NS-398, from Cayman Chemical Co.) for 15 minutes. After washes with warm $\left(37^{\circ} \mathrm{C}\right)$ medium, the cells were reincubated with fresh medium containing the same COX-1 and COX-2 inhibitors for 30 minutes. Cell supernatants were obtained by centrifugation at $10000 \mathrm{~g}$ at $4^{\circ} \mathrm{C}$ for 10 minutes and stored at $-70^{\circ} \mathrm{C}$ until assayed for $\mathrm{PGE}_{2}$ by a specific enzyme linked immunosorbent assay (ELISA; Biotrak, Amersham International, Slough, UK).

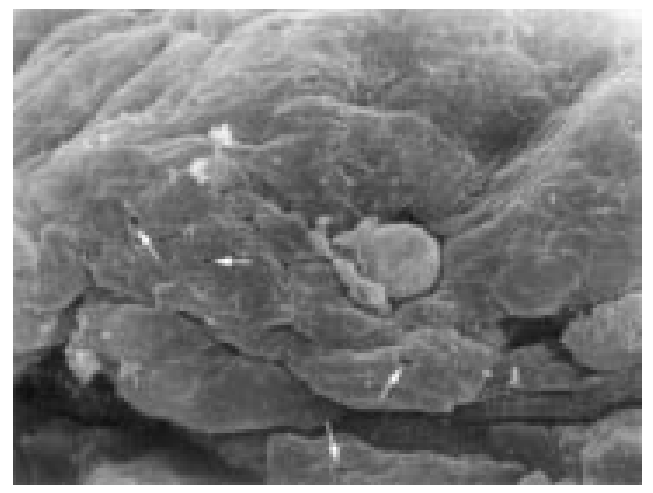

Figure 2 Scanning electron micrograph of a gastric mucosal sample denuded of epithelial cells. A number of small, discrete pores are present in the basement membrane, some of which are arrowed.

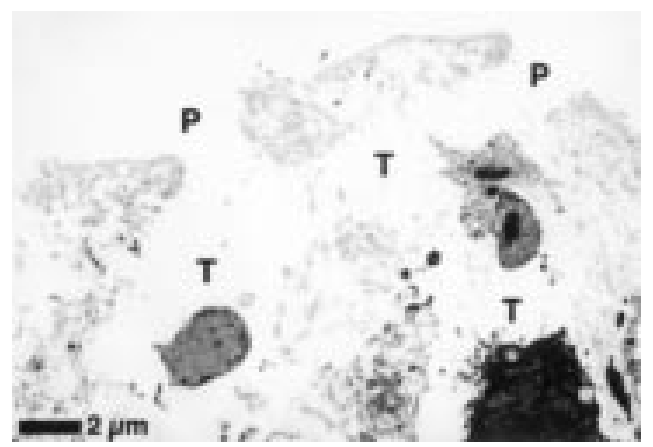

Figure 3 Transmission electron micrograph of a de-epithelialised gastric mucosal sample cultured for 24 hours. Discrete pores $(P)$ in the basement membrane are in continuity with tunnels $(T)$ or "channels" in the lamina propria matrix. A cell is present in the latter.

CULTURE FOR HELICOBACTER PYLORI

The presence of Helicobacter pylori was investigated by routine histological examination as well as culture of freshly isolated gastric mucosal samples. Mucosal samples were placed in sterile brucella broth with $15 \%$ glycerol (kept at $4^{\circ} \mathrm{C}$ ) and frozen at $-70^{\circ} \mathrm{C}$ within three hours. The samples were subsequently thawed and smeared across $7 \%$ horse blood agar plates and cultured for up to seven days, under microaerobic conditions (Campypak Plus, Becton Dickinson, Maryland, USA). Representative colonies from the plates were confirmed to be those from $H$ pylori by colony morphology and urease testing.

STATISTICAL ANALYSIS

Data are expressed as mean (SEM) and were analysed by analysis of variance and paired $t$ test.

\section{Results}

No $H$ pylori were seen in histological sections obtained from mucosal specimens used in this study. On culture of mucosal samples from seven of 11 gastric resection specimens, there was no growth of $H$ pylori.

Initial studies investigated the number of EDTA treatments required to remove surface epithelial cells completely. Following three sequential 30 minute incubations in $1 \mathrm{mmol} / 1$ EDTA, glandular epithelial cells were not completely removed (fig 1). Therefore, five sequential EDTA treatments were performed, 

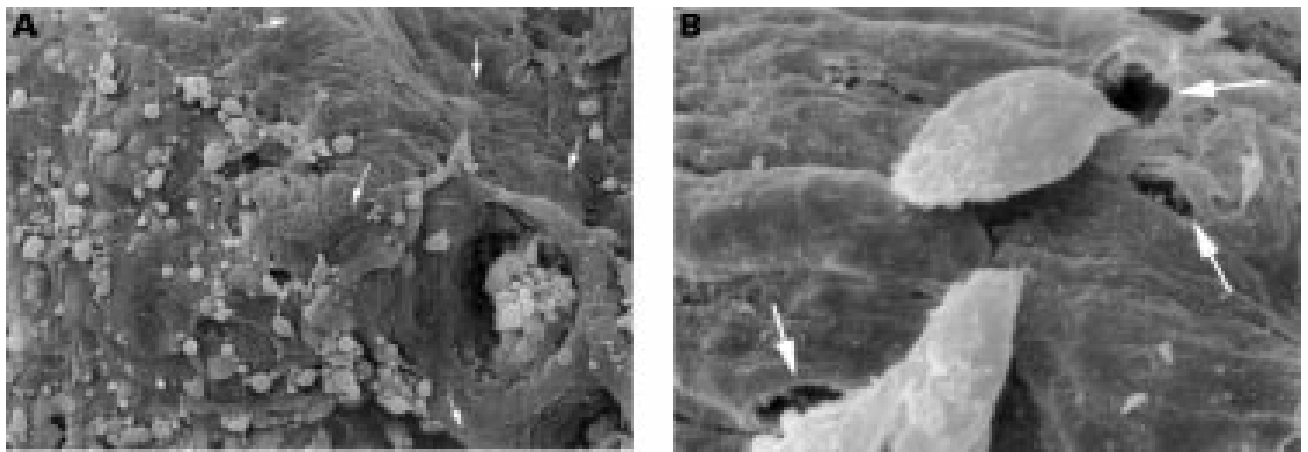

Figure 4 Low $(A)$ and high (B) power scanning electron micrographs of gastric mucosal samples denuded of epithelial cells and cultured for 24 hours. A number of discrete pores are present in the basement membrane (arrows), through which cells are migrating out of the lamina propria, as shown in (B).

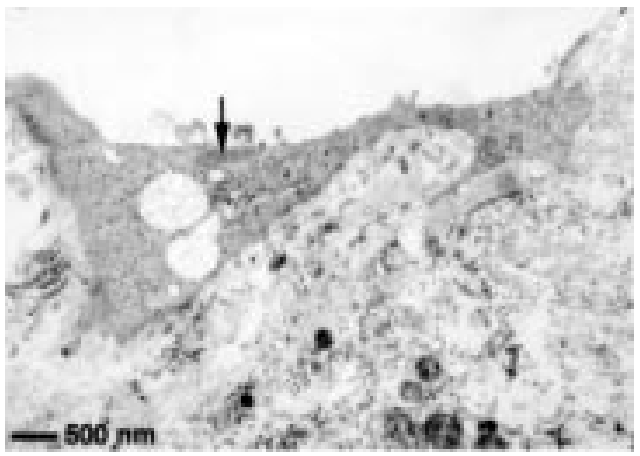

Figure 5 Transmission electron micrograph of a de-epithelialised gastric mucosal sample cultured for 72 hours. A myofibroblast, with bundle of microfilament (arrowed), is lying on the outer surface of the basement membrane.

when all the epithelial cells were consistently removed to leave an intact lamina propria, muscularis mucosae, and submucosa.

ELECTRON MICROSCOPY

Scanning electron microscopy of mucosal samples completely denuded of epithelial cells (before culture) showed the presence of numerous discrete pores in the basement membrane (fig 2). The diameter of the basement membrane pores was a mean 2.5 (SD 1.3) $\mu \mathrm{m}$ (range $0.65-8.29 \mu \mathrm{m}$; a total of 55 pores in three de-epithelialised mucosal samples were analysed). Transmission electron microscopy of denuded mucosal samples cultured for 24 hours showed the presence of "tunnels" in the lamina propria matrix which were in continuity with pores in the basement membrane (fig 3). During culture, cells were seen to be migrating out of the basement membrane pores (fig 4). After culture of denuded mucosal samples for 72 hours or more, myofibroblasts were often observed on the outer surface of the basement membrane (fig 5).

Table 1 Phenotype of cells migrating out of the lamina propria of de-epithelialised gastric mucosal samples

\begin{tabular}{lllll}
\hline & $\begin{array}{l}\text { T cells } \\
\text { (CD3+) }\end{array}$ & $\begin{array}{l}\text { B cells } \\
\text { (CD19+) }\end{array}$ & $\begin{array}{l}\text { Macrophages } \\
(\text { CD68+) }\end{array}$ & Eosinophils \\
\hline Day 1 (0-24 h) & $70.8(5.3)$ & $7.3(0.9)$ & $16.3(2.2)$ & $0.7(0.6)$ \\
Day 2 (24-48 h) & $69.6(5.7)$ & $6.3(0.9)$ & $12.5(2.4)$ & $1.6(0.6)$ \\
Day 3 (48-72 h) & $66.7(5.0)$ & $5.8(1.9)$ & $15.8(2.8)$ & $2.0(0.7)$ \\
\hline
\end{tabular}

Data are expressed as mean (SEM)\% of positive cells.

Cytospin preparations of cells (obtained from seven resection specimens) were analysed after immunohistochemical staining using specific antibodies.
CHARACTERISATION OF CELLS MIGRATING OUT OF THE GASTRIC LAMINA PROPRIA

Studies by phase contrast microscopy showed that during culture of gastric mucosal samples denuded of epithelial cells, cells began to appear in suspension and some were also seen adherent to the bottom of the culture dish. After 24 hours' culture, $1.4(0.3) \times 10^{6} \mathrm{cells} / \mathrm{g}$ tissue (viability $81.9(2.3) \%$ ) migrated out of the lamina propria and into the medium. Following reculture of the denuded mucosal samples in fresh medium, cells continued to migrate out of the lamina propria; cells/g tissue: 24-48 hour period of culture, $1.5(0.4) \times 10^{6}$ (viability $74.7(3.4) \%)$; 48-72 hours period of culture, $0.7(0.2) \times 10^{6}$ (viability $83.3(3.3) \%$ ). The number of cells migrating out of the lamina propria in the 48-72 hour period of culture was significantly smaller $(p<0.05)$ than that from the $0-24$ hour or $24-48$ hour periods of culture.

PHENOTYPIC ANALYSIS

The phenotype of the cells that had migrated out of the gastric lamina propria during culture for the first 72 hours, was analysed by immunohistochemistry (of cytospin preparations) and FACS analysis. Immunohistochemical studies showed that the migrating cells were mostly $\mathrm{T}$ cells and macrophages but also consisted of some B cells and eosinophils (table 1). Analysis of toluidine blue stained cytospin preparations showed only occasional polymorphonuclear cells (less than $1 \%$ in all). FACS analysis of gated lymphocytes showed that both CD 4 and CD8 positive T cells were present (table 2). The majority of the $\mathrm{T}$ cells expressed CD69 antigen and were CD48RO

Table 2 Phenotypic analysis of lymphocytes migrating out of the lamina propria of de-epithelialised gastric mucosal samples

\begin{tabular}{lll}
\hline & Day 1 & Day 2 \\
\hline CD19+ & $4.8(1.5)$ & $4.4(1.8)$ \\
CD3+ & $82.8(4.5)$ & $78.2(6.7)$ \\
CD4+ & $43.7(3.4)$ & $35.4(4.0)$ \\
CD8+ & $35.1(2.2)$ & $36.2(4.2)$ \\
CD16+56+ (CD3-) & $3.4(1.1)$ & $3.0(0.5)$ \\
CD3+CD16+56+ & $5.8(1.5)$ & $8.2(2.2)$ \\
CD3+CD25+ & $15.3(2.0)$ & $8.8(3.2)$ \\
CD3+CD69+ & $80.2(3.8)$ & $68.0(6.3)$ \\
CD45RO:RA ratio & $5.7(1.1)$ & $7.0(2.7)$ \\
\hline
\end{tabular}

Data are expressed as mean (SEM).

FACS analysis of gated lymphocytes was performed on cells obtained from seven resection specimens. 

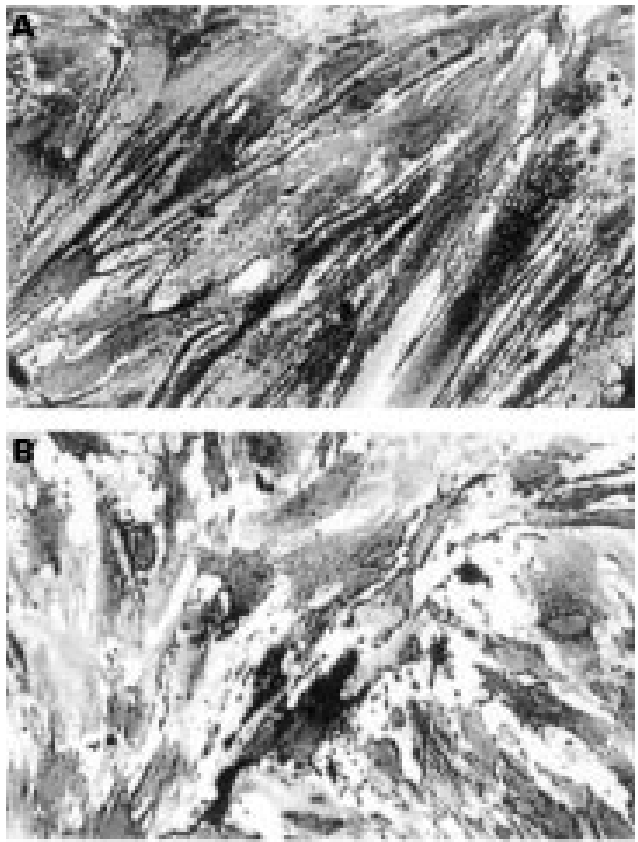

Figure 6 Expression of a smooth muscle actin $(A)$ and vimentin (B) by gastric myofibroblasts. The myofibroblasts were grown on coverslips and immunohistochemical labelling was performed using the relevant monoclonal antibodies. Cytoplasmically immunolabelled myofibroblasts are present in both $(A)$ and $(B)$.

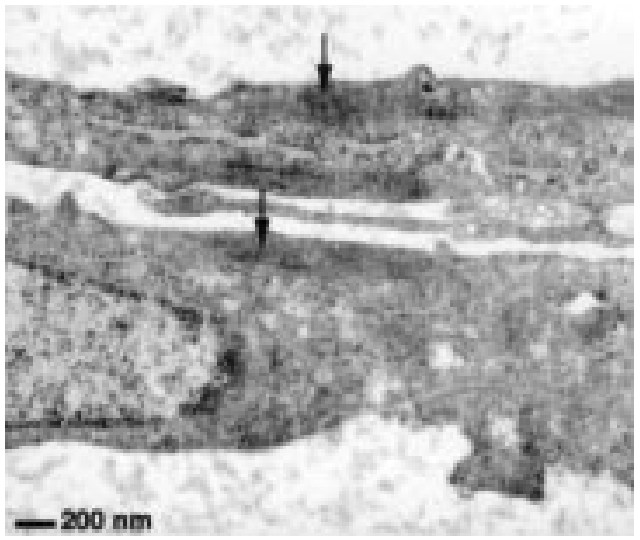

Figure 7 Transmission electron micrograph of cultured gastric myofibroblasts. Bundles of microfilament are arrowed.

positive. A significant proportion of cells also expressed CD25 (interleukin 2 receptor) and CD16/56. Only a small proportion of natural killer cells (CD16/56 positive, CD3 negative) was present. Consistent with findings on cytospin preparations, only a small proportion of $B$ cells was present.

CHARACTERISATION OF GASTRIC MYOFIBROBLASTS After culture of denuded mucosal samples for 72 hours or more, gastric myofibroblasts were frequently observed on the outer surface of the basement membrane (fig 5). After culture for seven to 10 days, myofibroblasts adherent to the culture dish began to appear and over the subsequent five to 10 days, colonies of myofibroblasts were seen, which gradually increased in size until a monolayer of myofibroblasts was formed. To date, myofibroblasts from denuded human gastric mucosa samples have been passaged nine times, some after freezing. The cells proliferate after seeding until a monolayer covers the culture flask; no overlapping myofibroblasts have been seen despite prolonged culture of confluent cells.

Immunocytochemical studies of gastric myofibroblasts at passages 1 and 8 showed that the cells express $\alpha$ smooth muscle actin (fig 6A) and vimentin (fig 6B) and were either weakly positive or negative for desmin (not shown). Electron microscopy studies showed that the gastric myofibroblasts expressed longitudinally arranged bundles of microfilaments below the cell membrane (fig 7).

EXPRESSION OF COX-1 AND COX-2

Studies by RT-PCR showed that the gastric myofibroblasts express mRNA transcripts for COX-1 and COX-2 enzymes (fig 8). The presence of COX-1 and COX-2 protein was confirmed by immunohistochemistry (fig 9).

Functional activity of the two isoforms of COX enzyme in gastric myofibroblasts was shown by inhibition of $\mathrm{PGE}_{2}$ release in the

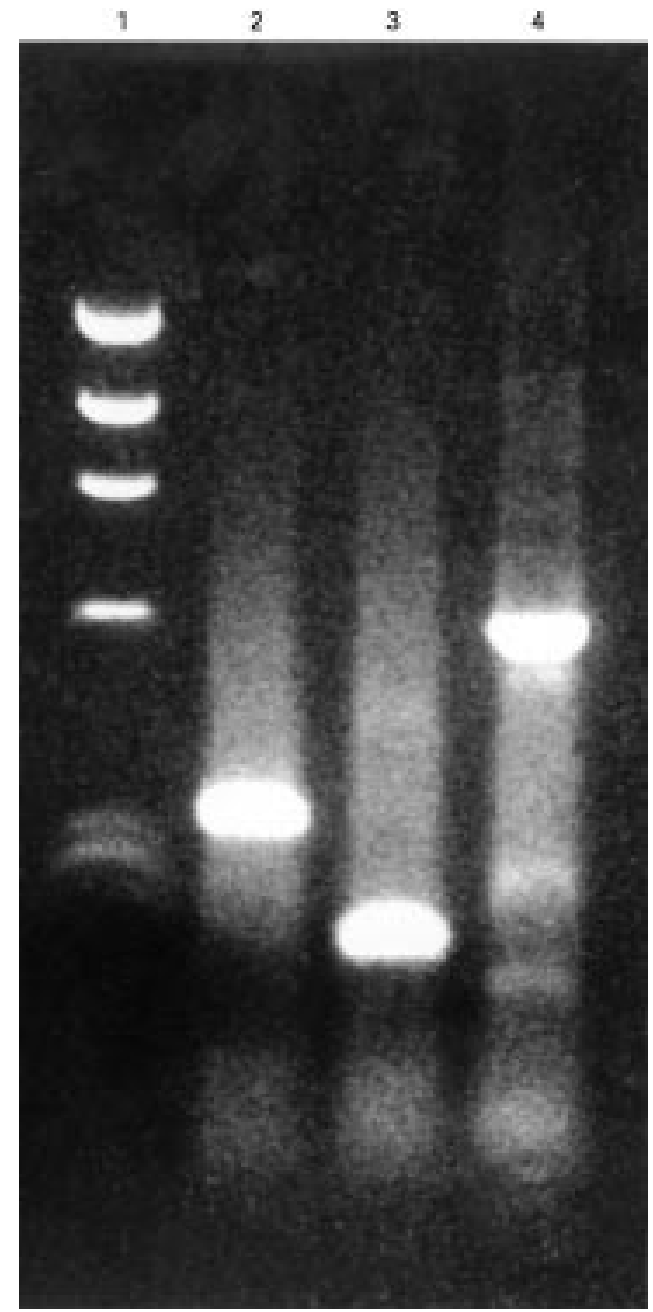

Figure 8 Gastric myofibroblasts express $m R N A$ transcripts for COX-1 and COX-2. RNA was isolated from a confluent monolayer of myofibroblasts, reverse transcribed, and relevant transcripts were amplified by polymerase chain reaction using specific primers. Lane 1, DNA size markers; lane 2, COX-1 product; lane 3, COX-2 product; lane 4, glyceraldehyde-3-phosphate dehydrogenase (GAPDH) product. 


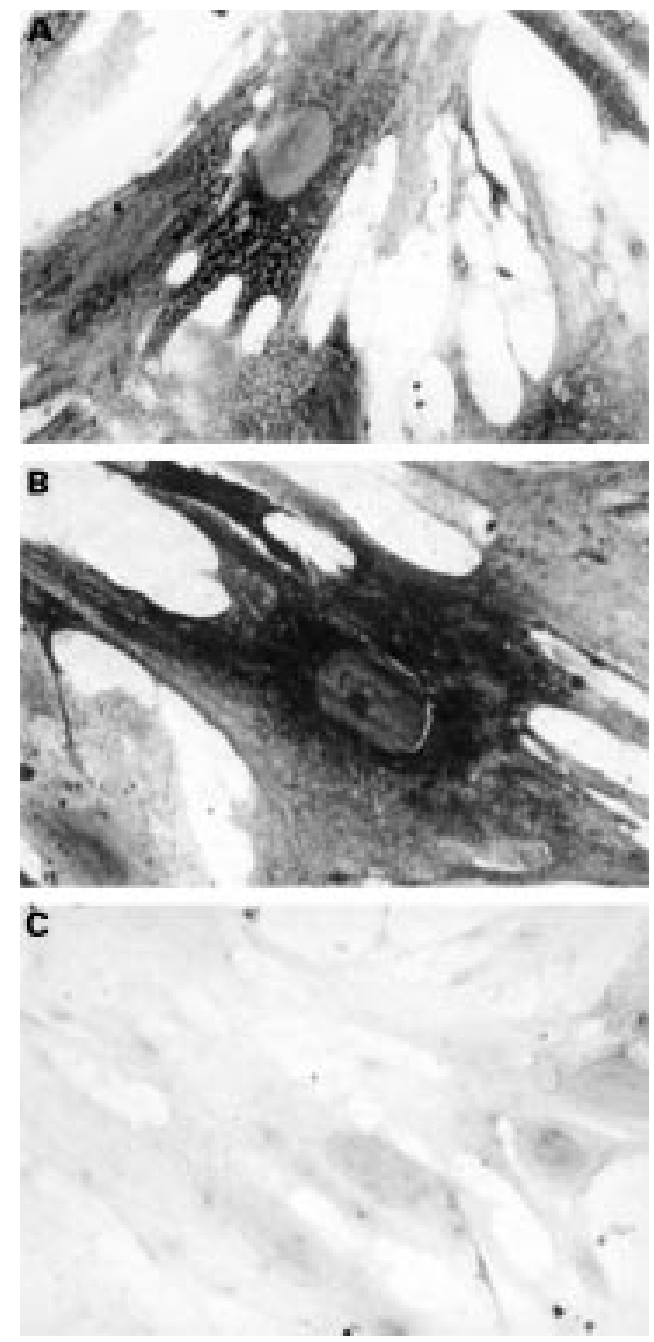

Figure $9 \quad C O X-1$ (A) and $C O X-2$ (B) immunoreactivity in gastric myofibroblasts. Confluent monolayers of myofibroblasts grown on coverslips were used for immunohistochemical studies using antibodies to COX-1 (A), COX-2 (B), or control buffer instead of the primary antibody $(C)$. Strong COX-2 immunoreactivity is seen in the perinuclear region in $(B)$.

presence of selective COX-1 (SC-58560) and COX-2 (NS-398) inhibitors ( $\mathrm{PGE}_{2}$ release as percentage of control medium: $10^{-7} \mathrm{M}$ SC58560, $36.5(10.6) \% ; 10^{-7} \mathrm{M}$ NS-398, 43.3 (11.9) $\%$; p <0.01 compared with control for both). When cultured with control medium, the myofibroblasts released 315 (80) pg/well $\mathrm{PGE}_{2}$.

Twenty four hour culture supernatants of lamina propria cells (predominantly lymphocytes and macrophages, cultured at $2 \times 10^{6}$ cells $/ \mathrm{ml}$ ), obtained following 24 hour culture of denuded gastric mucosal samples, contained 11.6 (4.9) ng/ml PGE $(\mathrm{n}=4)$. In cytospin preparations of these cells, mononuclear cells with morphological features of macrophages were labelled by antibodies to COX-1 and COX-2. The macrophage phenotype of these cells was confirmed by their capacity to phagocytose opsonised zymosan (fig 10).

\section{Discussion}

In this study, we have shown that the subepithelial basement membrane of gastric mucosa contains discrete pores ranging in

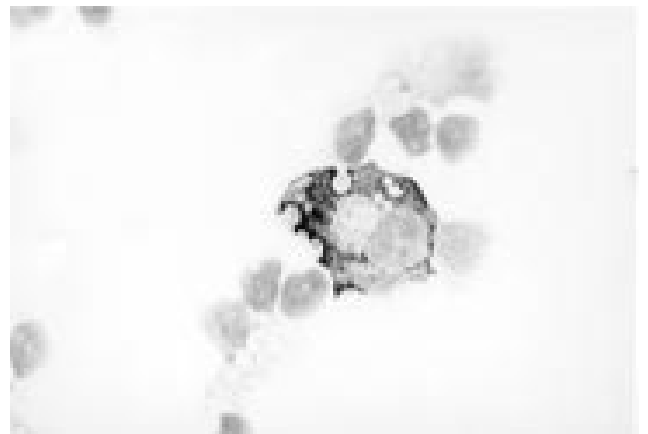

Figure 10 Expression of COX-2 by gastric lamina propria macrophages. Lamina propria mononuclear cells were cultured with opsonised zymosan and cytospin preparations of the cells subsequently labelled for COX-2 by immunohistochemistry. A COX-2 immunoreactive macrophage, containing a number of phagocytosed zymosan articles is seen. Other cells (mainly lymphocytes) are negative.

diameter from 0.65 to $8.3 \mu \mathrm{m}$. When gastric mucosal samples denuded of epithelial cells were placed in culture, large numbers of cells migrated out of the lamina propria via the basement membrane pores that are in continuity with "tunnels" or channels in the underlying matrix. During the first 72 hours of culture of the denuded mucosal samples, the migrating cells were mostly lymphocytes and macrophages. The lymphocytes were predominantly $\mathrm{T}$ cells (CD4+ or CD8+) and the majority of them expressed CD45RO suggesting that they are "memory" $\mathrm{T}$ cells. ${ }^{12}{ }^{13}$ The majority of gastric lamina propria $\mathrm{T}$ cells also expressed CD69, which is a $28-32 \mathrm{kDa}$ homodimer that is not present on the surface of resting $\mathrm{T}$ cells but is rapidly and transiently expressed on activation in vitro. ${ }^{14}{ }^{15}$ Thus, expression of CD69 by a majority of gastric lamina propria $\mathrm{T}$ cells suggests that they are continuously stimulated by antigen, likely to be derived from the lumen. $H$ pylori in the mucus layer can be a source of such antigens. However, the mucosal samples used in our study did not show inflammation or the presence of $H$ pylori on histological examination. Moreover, $H$ pylori was not grown on culture of many of the mucosal samples. Consistent with the lack of inflammation on histological examination, neutrophils were virtually absent from the migrating lamina propria cell population. Thus the CD69 expressing gastric lamina propria $\mathrm{T}$ cells are likely to be stimulated by antigens other than those derived from $H$ pylori, present in the normal gastric luminal contents. The majority of the normal colonic lamina propria $\mathrm{T}$ cells have also been shown to express CD $69,{ }^{16}$ suggesting that lamina propria $\mathrm{T}$ cells throughout the gastrointestinal tract are continuously stimulated.

Among the cells migrating out of the denuded gastric mucosal samples over 72 hours, a significant proportion of macrophages was also consistently seen. They expressed COX-1 and COX-2 enzymes and are likely to be the source of $\mathrm{PGE}_{2}$ secreted by the lamina propria cells obtained after 24 hours of culture of denuded gastric mucosal samples. In studies on tissue sections of normal and ulcerated gastric mucosal samples, we have recently shown that lamina propria macrophages express 
immunoreactive COX-1 and COX-2 enzymes. ${ }^{17}$ In the present study, by showing their capacity to phagocytose opsonized zymosan, we have confirmed that COX-1 and COX-2 expressing lamina propria mononuclear cells are macrophages (in addition to myofibroblasts, see below).

After culture of denuded gastric mucosal samples for 72 hours or more, myofibroblasts were frequently observed on the outer surface of the basement membrane. Subsequently, colonies of myofibroblasts adherent to the culture dish began to appear, which grew to become confluent monolayers of cells that appeared homogenous by phase contrast microscopy. These cells were confirmed to be myofibroblasts as shown by expression of features of both fibroblasts and smooth muscle cells. ${ }^{6}{ }^{18}$ The gastric myofibroblasts strongly expressed $\alpha$ smooth muscle actin and were positive for vimentin. Ultrastructurally, they contained bundles of microfilament close to the cell membrane. We have recently also shown the migration and establishment in culture of human colonic subepithelial myofibroblasts. The gastric myofibroblasts show similar morphological features and like their counterparts from the colon, retain a representative and differentiated phenotype despite prolonged culture and passage. Furthermore, in common with the colonic cells, the gastric myofibroblasts express mRNA, protein, and bioactivity for COX-1 and COX-2 enzymes. We have recently shown that subepithelial myofibroblasts in sections of normal gastric mucosa express immunoreactive COX-1 and COX-2 enzymes. ${ }^{17}$ Because of their presence subjacent to the basement membrane, the myofibroblasts are likely to be able to influence the function of overlying epithelial cells. Myofibroblast derived secretory factors, such as products of COX-1 and COX-2 enzymes, would be capable of interacting with the basal surface of overlying epithelial cells via pores in the basement membrane.

Normal colonic myofibroblasts synthesise the extracellular matrix proteins collagen type IV, fibronectin, and laminin $\beta 1$ and $\gamma 1 .^{6}$ In recent studies, they have been shown to be capable of modulating transepithelial resistance and secretory response of intestinal epithelial cells, ${ }^{19}{ }^{20}$ enhance epithelial restitution, ${ }^{21}$ induce morphological and cytological differentiation in T84 colonic epithelial cells, ${ }^{22}$ and regulate epithelial cell proliferation. ${ }^{23}$ The functions of gastric myofibroblasts remain to be characterised. They may be different from intestinal myofibroblasts as such cells isolated from different regions of the rat intestine display regional characteristics. ${ }^{24}$ As we have shown that the isolated gastric myofibroblasts retain a representative and differentiated phenotype, despite prolonged culture and passage, they should be suitable for functional studies of interactions with other lamina propria cells and epithelial cells.

In our studies, $H$ pylori negative gastric mucosal samples were used. We postulate that in $H$ pylori infected gastric mucosa, polymorphonuclear cells, lymphocytes, and macro- phages would be capable of migrating, via basement membrane pores, into the epithelium and the mucus layer. Surface proteins from $H$ pylori have been shown to exhibit chemotactic activity for these cells, ${ }^{25}$ which would be capable of interacting directly with the bacteria in the mucus layer. We also postulate that following the loss of injured epithelial cells, $H$ pylori or its products would be capable of gaining access into the exposed lamina propria, via basement membrane pores. Such access to the lamina propria may occur before epithelial continuity and barrier function is reestablished via migration of adjacent viable cells. ${ }^{26}$ Our present studies suggest that lamina propria cells (lymphocytes macrophages and myofibroblasts) obtained during culture of denuded gastric mucosal samples would be suitable for studies of their interactions with $H$ pylori and its products.

The electron microscopy studies used equipment funded by the Wellcome Trust.

1 Castro GA, Arntzen CJ. Immunophysiology of the gut: a research frontier for integrative studies of the common mucosal immune system. Am f Physiol 1993;265:G599610.

2 Perdue $\mathrm{MH}, \mathrm{McKay} \mathrm{DM}$. Integrative immunophysiology in the intestinal mucosa. Am f Physiol 1994;267:G151-65.

De Witt D, Smith WL. Yes, but do they still get headaches? Cell 1995;83:345-8.

4 Low FN, McClugage SG. Microdissection by ultrasonication: scanning electron microscopy of the ultrasonication: scanning electron microscopy of the Am f Anat 1984;169:137-47.

5 Mahida YR, Galvin A, Gray T, et al. Migration of human intestinal lamina propria lymphocytes, macrophages and eosinophils following the loss of surface epithelial cells. Clin Exp Immunol 1997;109:377-86.

6 Mahida YR, Beltinger J, Makh S, et al. Adult human colonic subepithelial myofibroblasts express extracellular matrix proteins and cyclooxygenase-1 and -2. Am f Physiol 1997; 273:G1341-8.

7 Mahida YR, Wu K, Patel S, et al. Respiratory burst activity of intestinal macrophages in normal and inflammatory bowel disease. Gut 1989;30:1362-70.

8 Pallis M, Robins A, Powell R. Quantitative analysis of lymphocyte CD11a using standardised flow cytometry. Scand 7 Immunol 1993;38:558-64.

9 Robinson G, Gray T. Electron microscopy 2. Tissue preparation, sectioning and staining. In. Bancroft JD, Stevens A, eds. Theory and practice of histological techniques. 3rd edn. eds. Theory and practice of histological techniques.

10 Diaz A, Reginato AM, Jimenez SA. Alternative splicing of human prostaglandin $\mathrm{G} / \mathrm{H}$ synthase mRNA and evidence of differential regulation of the resulting transcripts by ransforming growth factor $\beta 1$, interleukin $1 \beta$ and tumor necrosis factor $\alpha$. F Biol Chem 1992;267:10816-22.

11 Hla T, Neilson K. Human cyclooxygenase-2 cDNA. Proc Natl Acad Sci USA 1992;89:7384-8.

2 Sanders ME, Makgoba MW, Shaw S. Human naive and memory $\mathrm{T}$ cells: reinterpretation of helper-inducer and suppressor-inducer subsets. Immunol Today 1988;9:195-9.

13 James SP, Zeitz M. Human gastrointestinal mucosal T cells. In: Ogra PL, Lamm ME, McGhee JR, et al, eds. Handbook of mucosal immunology. San Diego: Academic Press, 1994:275-85.

14 Hara T, Jung LKL, Bjorndahl JM, et al. Human T cell activation III. Rapid induction of a phosphorylated $28 \mathrm{kd} / 32$ $\mathrm{kD}$ disulfide-linked early activation antigen (EA 1) by $\mathrm{kD}$ disulfide-linked early activation antigen (EA 1) by 12-O-tetradecanoyl phorbol-13-acetate,
tigens. F Exp Med 1986;164:1988-2005.

15 Testi R, Phillips JH, Lanier LL. Constitutive expression of a Testi R, Phillips JH, Lanier LL. Constitutive expression of a
phosphorylated activation antigen (Leu 23) by CD (bright) human thymocytes. F Immunol 1988;141:2557-63.

16 De Maria R, Fias S, Silvestri M, et al. Continuous in vivo activation and transient hyporesposiveness to TcR/CD3 triggering of human gut lamina propria lymphocytes. Eur $\mathcal{F}$ Immunol 1993;23:3104-8.

17 Wu KC, Jackson L, Mahida YR, et al. Expression of cyclooxygenase (COX)-1 and -2 in normal and ulcerated human gastric mucosa [abstract]. Gut 1997;41(suppl 3):A146.

18 Valentich JD, Powell DW. Intestinal subepithelial myofibroblasts and mucosal immunophysiology. Curr Opin Gastroenterol 1994;10:645-51.

19 Beltinger J, Makh S, Stack WA, et al. Human colonic subepithelial myofibroblasts modulate transepithelial resubepithelial myofibroblasts modulate transepithelial re-
sistance and secretory response in epithelial cells [abstract]. Gut 1997;41(suppl 3):A29.

20 Hinterleitner TA, Saada JI, Berschneider HM, et al. IL-1 stimulates intestinal myofibroblast COX gene expression 
and augments activation of $\mathrm{Cl}^{-}$secretion in T84 cells. $A m \mathcal{F}$ Physiol 1996;271:C1262-8.

21 McKaig B, Makh S, Hawkey CJ, et al. Human intestinal subepithelial myofibroblasts stimulate repair of wounded intestinal epithelial monolayers [abstract]. Gut 1997; 41(suppl 3):A29.

22 Del Buono R, Beltinger J, Jackson L, et al. Primary adult human colonic subepithelial myofibroblasts induce morphological and cytological differentiation in T84 colonic epithelial cells [abstract]. Gut 1998; 42(suppl 1):A89

23 Göke M, Kanai M, Podolsky DK. Intestinal fibroblasts regulate intestinal epithelial cell proliferation via hepatocyte growth factor. Am F Physiol 1998;274:G809-18.

4 Plateroti M, Rubin DC, Duluc I, et al. Subepithelial fibroblast cell lines from different levels of gut axisdisplay regional characteristics. Am 7 Physiol 1998;274:G945-54.

25 Mai UEH, Perez-Perez GI, Allen JB, et al. Surface proteins from Helicobacter pylori exhibit chemotactic activity for human leukocytes and are present in gastric mucosa. $\mathcal{F}$ Exp Med 1992;175:517-25.

26 Lacy ER. Epithelial restitution in the gastrointestinal tract. $\mathcal{F}$ Clin Gastroenterol 1988;10(suppl 1):72-7. 